\title{
Testing the scalar expectancy theory (SET) and the learning-to-time model (LeT) in a double bisection task
}

\author{
ARMANDO MACHADO and PAULO PATA \\ University of Minho, Braga, Portugal
}

\begin{abstract}
Two theories of timing, scalar expectancy theory (SET) and learning-to-time (LeT), make substantially different assumptions about what animals learn in temporal tasks. In a test of these assumptions, pigeons learned two temporal discriminations. On Type 1 trials, they learned to choose a red key after a 1-sec signal and a green key after a 4-sec signal; on Type 2 trials, they learned to choose a blue key after a 4-sec signal and a yellow key after either an 8-sec signal (Group 8) or a 16-sec signal (Group 16). Then, the birds were exposed to signals $1 \mathrm{sec}, 4 \mathrm{sec}$, and $16 \mathrm{sec}$ in length and given a choice between novel key combinations (red or green vs. blue or yellow). The choice between the green key and the blue key was of particular significance because both keys were associated with the same 4-sec signal. Whereas SET predicted no effect of the test signal duration on choice, LeT predicted that preference for green would increase monotonically with the length of the signal but would do so faster for Group 8 than for Group 16. The results were consistent with LeT, but not with SET.
\end{abstract}

The study of temporal discrimination in animals has undergone substantial progress during recent decades. On the empirical side, researchers have devised several procedures for studying timing and have obtained some robust findings with them (for reviews, see Gallistel, 1990; Gallistel \& Gibbon, 2002; Shettleworth, 1998; Staddon, 2001). On the theoretical side, they have advanced several quantitative models to explain the data, including scalar expectancy theory (SET) model, an informationprocessing model developed by Gibbon $(1977,1991)$, and the learning-to-time (LeT) model, a behavioral model developed by Machado (1997) on the basis of earlier work by Killeen and Fetterman (1988). In the present study, we report an experiment that was designed to contrast the predictions of these two timing models.

Consider the temporal bisection procedure. A pigeon experiences one of two stimuli-for example, a short- or a long-duration light - and then it chooses between two keys, one red and the other green. The choice of red is correct (and therefore rewarded) following the short signal, and the choice of green is correct following the long one. After the animal learns the discrimination, the experimenter presents it with test signals of intermediate duration and records the animal's preference for, say, the green key. It is commonly found that as the duration of

The research reported in this article was supported by a grant to A.M. from the Portuguese Foundation for Science and Technology. The authors thank students Joana Arantes and António Fidalgo for their help in running the experiment. Correspondence concerning this article should be addressed to A. Machado, Instituto de Educação e Psicologia, Universidade do Minho, 4710 Braga, Portugal (e-mail: armandom@iep. uminho.pt). the test stimulus increases from $1 \mathrm{sec}$ to $4 \mathrm{sec}$, the preference for the green key increases monotonically from about 0 to about 1 . Typically, this psychometric function equals .5 (the point of subjective equality) at about the geometric mean of the short and long signals (see, e.g., Catania, 1970; Church \& Deluty, 1977; Fetterman \& Killeen, 1991; Platt \& Davis, 1983; Stubbs, 1968).

How do SET and LeT explain these findings? SET postulates an internal clock, whose structure is represented in the left panel of Figure 1: A pacemaker generates pulses at a high rate; an accumulator counts the pulses emitted during the interval to be timed; and a long-term memory store saves the count obtained at the end of the interval. In the temporal bisection task, SET assumes that the animal forms two memory stores during training, one containing the counts obtained at the end of the short stimulus and the other, the counts obtained at the end of the long stimulus. To decide which key to choose at the end of a signal, the animal compares the number that is in the accumulator when the signal ends (i.e., the number of pulses generated during the signal, $X_{\mathrm{T}}$ ) against two samples, one extracted from the memory store for short durations, $X_{\mathrm{S}}$, and the other from the memory store for long durations, $X_{\mathrm{L}}$. If the ratio $X_{\mathrm{S}} / X_{\mathrm{T}}$ is greater than the ratio $X_{\mathrm{T}} / X_{\mathrm{L}}$, then the number is "closer" to the sample extracted from the short stimulus store and the animal is more likely to choose the red key. During testing, as the signal duration increases from short to long, $X_{\mathrm{T}}$ increases from values close to $X_{\mathrm{S}}$ to values close to $X_{\mathrm{L}}$; hence, the probability of choosing the green key increases with signal duration (see Gibbon, 1981; also see Gallistel, 1990).

LeT, on the other hand, consists of three major components (see the right panel of Figure 1): a serial organi- 
SET

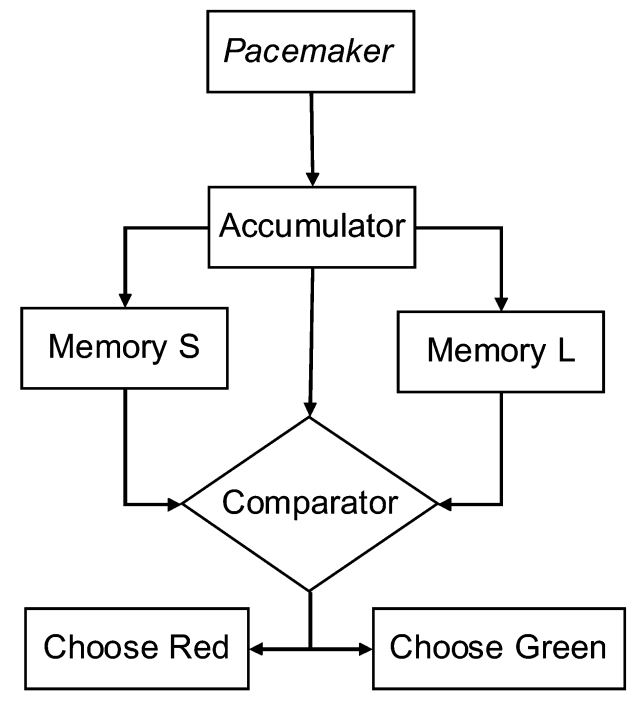

LeT

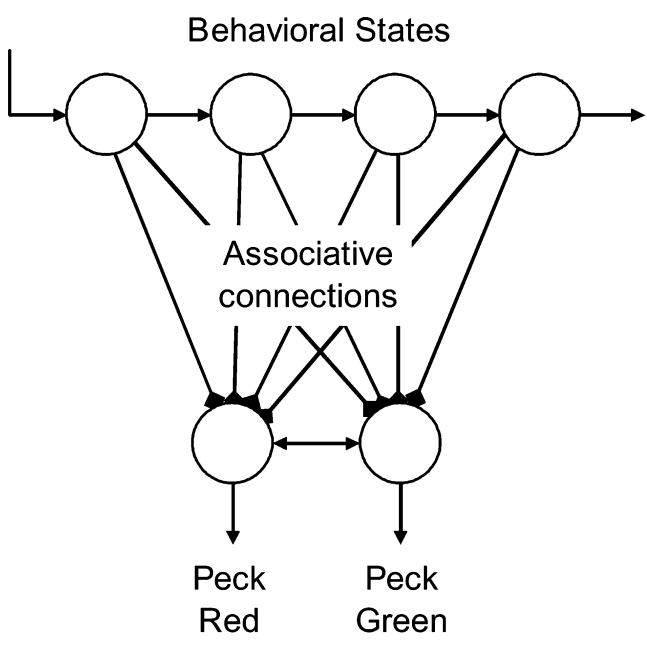

Figure 1. Left: Structure of the scalar expectancy theory (SET) model for the bisection procedure. A pacemaker generates pulses, which are accumulated in an accumulator. Two memory stores save the counts obtained at the end of the short $(S)$ and long $(L)$ signals. To decide which key to choose, the animal compares the number that is in the accumulator when the test signal ends against two samples, one extracted from each memory store. If the count in the accumulator is closer to that of the sample extracted from the short memory store, then the animal is more likely to choose the $S$ key. Right: Structure of the learning-to-time (LeT) model. After a time marker, a series of behavioral states (top circles) is activated. The states may be coupled to various degrees (middle connections) with the two instrumental responses (bottom circles). The strength of each response is determined by the dot product between the vectors of state activation and coupling.

zation of behavioral states, a vector of associative links connecting the behavioral states to the instrumental responses, and the instrumental responses themselves. At the onset of the interval to be timed, only the first state is active, but as time elapses the activation spreads from each state to the next state in the series. Each behavioral state is also coupled with the instrumental responses, and the degree of coupling changes with training, decreasing during extinction and increasing during reinforcement. The strength of an instrumental response at a given moment depends on which state is the most active at that moment and on how strong the associative link is between that state and the response. Applied to the bisection task, LeT predicts that after the short stimulus only the early states of the cascade are active and, because the choice of the red key is rewarded whereas that of the green key is extinguished, those early states will be coupled strongly with the red, or "short," response and weakly with the green, or "long" response. Conversely, following the long stimulus the later states are the most active, and because the choice of green is rewarded whereas the choice of red is extinguished, those later states will be coupled strongly with the green, or "long," response and weakly with the red, or "short" response. During testing, as signal duration increases, the state most active at the moment of choice also increases. Because earlier states are coupled mostly with the red, "short" response whereas later states are coupled mostly with the green, "long" response, the probability of choosing "long" increases with signal duration.

Although the two models differ in their conceptualization of temporal learning, both predict monotonically increasing psychometric functions with the point of subjective equality at (SET) or approximately at (LeT) the geometric mean of the training stimuli. To separate the predictions of SET and LeT, Machado and Keen (1999) modified the task just described. Assume that a pigeon has learned not one but two temporal discriminations within the same session (see Figure 2, top panel). In the presence of two keys, one red and one green, the choice of red is rewarded if the preceding signal was $1 \mathrm{sec}$ long and the choice of green is rewarded if the signal was $4 \mathrm{sec}$ long. We call these trials Type 1 trials, and, because signal duration and key color are correlated, we refer to the correct choices following the 1-sec and 4-sec signals as $\mathrm{S} 1$ and L1, respectively. In the presence of two other keys, one blue and the other yellow, the choice of blue is rewarded following a 4-sec signal, whereas the choice of yellow is rewarded following a 16-sec signal. We call these trials Type 2 trials and refer to the correct choices following the 4-sec and 16-sec signals as S2 and L2, respectively. Thus, S1 and L1 are the "short" and "long" 


\section{TRAINING}

Trial Type 1

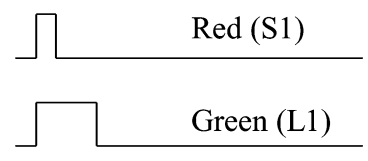

Trial Type 2

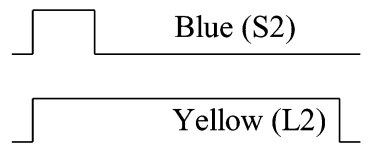

\section{TESTING}

Signal Durations

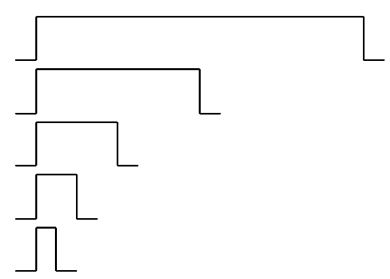

Choice keys

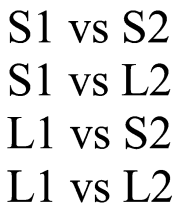

Figure 2. Structure of a double bisection procedure during training and testing. Top: On Type 1 trials, a 1-sec or a 4-sec signal occurs. A red key choice (S1) is rewarded if the signal was 1 sec long, and a green key choice (L1) is rewarded if it was 4 sec long. On Type 2 trials, the signal is either 4 sec or 16 sec long. After the former, the choice of blue (S2) is rewarded; after the latter, the choice of yellow (L2) is rewarded. Bottom: During testing, the signal ranges from 1 to 16 sec; then the animal is given a choice between two keys that it has not seen together before.

choices in the first range of durations, and S2 and L2 are the "short" and "long" choices in the second range of durations. Note that the 4-sec duration is associated with both L1 and S2. What, then, will a bird do when, following a $t$-sec signal, it is presented with a pairing of keys that it has never seen before (see Figure 2, bottom panel)?

Consider, for example, the choice between the green and blue keylights (L1 and S2, respectively). According to SET, the animal should always be indifferent between them because in the presence of these keylights the just experienced test duration would be compared with two samples that came from identical distributions (i.e., L1 and $\mathrm{S} 2$ are both associated with an experienced duration of $4 \mathrm{sec}$ ). This prediction is illustrated by the horizontal line in the left panel of Figure 3. The important feature of SET's prediction is not the specific value of the preference for L1 - bias for one color, for example, could naturally change that value - but the absence of any relation between preference for L1 and the duration of the test signal.
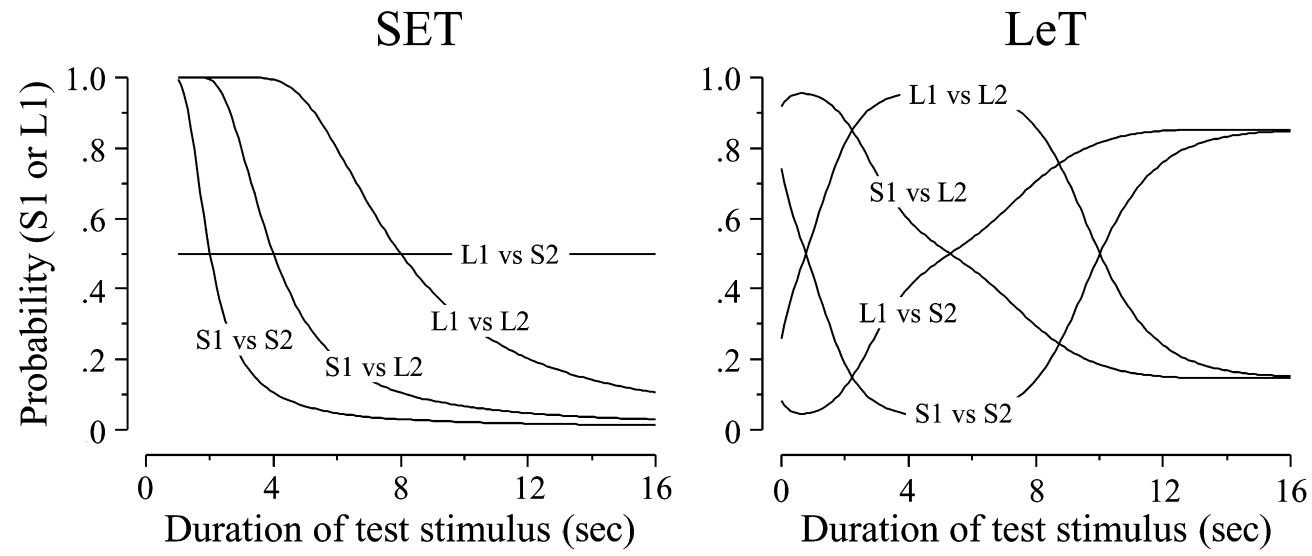

Figure 3. Predictions of the scalar expectancy theory (SET) model and the learning-to-time (LeT) model for test trials with new key combinations (see the Appendix for mathematical derivations). The SET curves use one parameter: $\gamma=0.4$. The LeT curves use three parameters: $\lambda=2, \alpha=1$, and $\delta=3.5$. 
Table 1

Relative Strength of the Couplings Between the Behavioral States and the Choice Responses Predicted by LeT

\begin{tabular}{cccc}
\hline \multirow{2}{*}{$\begin{array}{c}\text { Choice } \\
\text { Response }\end{array}$} & Bnitial & Middle & Final \\
\cline { 2 - 4 } & ++ & 0 & + \\
S1 & 0 & ++ & + \\
L1 & + & ++ & 0 \\
S2 & + & 0 & ++ \\
L2 & & 0 & \\
\hline
\end{tabular}

Note-The behavioral states are divided into three classes: initial (states most active at $1 \mathrm{sec}$ ), middle (states most active at $4 \mathrm{sec}$ ), and final (states most active at $16 \mathrm{sec}$ ). The symbols,+++ , and 0 denote relatively strong, moderate, and weak couplings, respectively.

In contrast, LeT predicts that the animal's preference for L1 will increase with the duration of the signal (see the L1-vs.-S2 curve in the right panel of Figure 3). In the Appendix, we derive LeT's prediction mathematically; here we give a less rigorous but more intuitive account. We divide the behavioral states into three classes: the initial states, which are the most active at $1 \mathrm{sec}$; the middle states, which are the most active at $4 \mathrm{sec}$; and the final states, which are the most active at $16 \mathrm{sec}$. We also let the symbols $0,+$, and ++ stand for weak, moderate, and strong couplings between a class of states and a response, respectively.

The model's predictions derive from the profile of couplings learned during training, and these profiles are shown in Table 1. Thus, during Type 1 trials the initial states become coupled with S1 and uncoupled with L1, whereas the middle states become coupled with L1 and uncoupled with S1. Final states do not become active during Type 1 trials and therefore do not change their initial (equal and moderate) couplings with S1 and L1. During Type 2 trials, the middle states become coupled with S2 and uncoupled with L2, whereas the final states become coupled with L2 and uncoupled with S2; the initial states retain their initial (equal and moderate) couplings with S2 and L2. To predict the animal's choices when L1 and S2 are presented together during test trials, look at the rows corresponding to responses L 1 and S2 in Table 1 and consider the following cases: (1) If the test stimulus is $1 \mathrm{sec}$ long, then the initial states will be the most active at the moment of choice and, because they are coupled less with L1 than with S2, the animal is unlikely to choose L1; (2) if the test stimulus is 4 sec long, then the middle states will be the most active at the moment of choice and, because they are equally coupled with L1 and S2, the animal is equally likely to choose L1 or S2; and (3) if the test stimulus is $16 \mathrm{sec}$ long, then the final states will the most active at the moment of choice and, because they are coupled more with L1 than with S2, the animal is likely to choose L1. That is, as the duration of the signal increases, the probability of choosing L1 also increases.

The remaining curves in Figure 3 show that SET and LeT also make different predictions for the other three key combinations. Whereas the shape of the curves is always the same for SET (the three curves are scale transforms of one another), LeT predicts a U-shaped curve when the choice is between S1 and S2, an inverted Ushaped curve when the choice is between L1 and L2, and a descending curve when the choice is between S1 and L2. (The general trend of these predictions is readily understood from Table 1). In fact, LeT predicts that the two pairs of curves - S1 versus L2 and L1 versus S2 on the one hand, and L1 versus L2 and S1 versus S2 on the other hand-will be symmetric around the indifference line $y=.5$ (see the Appendix for quantitative details).

Machado and Keen's (1999) results were always closer to LeT's predictions than to those of SET. In particular, they found that when a critical choice was to be made between L1 and S2, preference for L1 increased monotonically with stimulus duration. Because this result seriously puts into question the dominant model of timing in the field (i.e., SET), it needs to be reproduced. This was the first goal of the present study.

The two models make another set of different predictions for this task. Assume that one group of pigeons learns the two discriminations mentioned above (" 1 versus 4" and "4 versus 16") whereas another group learns a slightly different pair of discriminations ("1 versus 4 " and " 4 versus 8 "). That is, the groups differ only in the longer signal duration. To emphasize this fact, we refer to these two groups as Group 16 and Group 8, respectively. How will the two groups perform during the test trials with the four key combinations?

Figure 4 shows what the models predict for the four cases. First, both models predict that when a choice must be made between $\mathrm{S} 1$ and L2, preference for S1 will decrease with stimulus duration, but more rapidly for Group 8 than for Group 16. Second, when a choice must be made between L1 and S2, SET predicts no effect of stimulus duration. In contrast, LeT predicts that preference for L1 will increase with stimulus duration, but more rapidly for Group 8 than for Group 16. Third, when the choice is between $\mathrm{S} 1$ and $\mathrm{S} 2$, SET predicts the same monotonically decreasing function for the two groups, whereas LeT predicts two distinct U-shaped functions. The function for Group 16 should be wider than the function for Group 8. Fourth, SET predicts that when the choice is between L1 and L2, preference for L1 should decrease with stimulus duration, but more rapidly for Group 8 than for Group 16. LeT predicts two inverted Ushaped functions, with the function for Group 16 being wider than that for Group 8.

To understand intuitively why LeT predicts different curves for Groups 8 and 16, particularly in the critical case of L1 versus S2, consider the following. In both groups, the profile of couplings between the behavioral states and L1 will be identical because the groups do not differ on the Type 1 trials. However, the profile of couplings with S2 will differ because the groups differ on the Type 2 trials. Specifically, in Group 8 the states most active at $8 \mathrm{sec}$ will be uncoupled with S2, and conse- 
SET

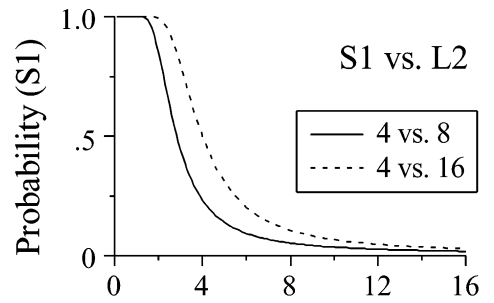

LeT
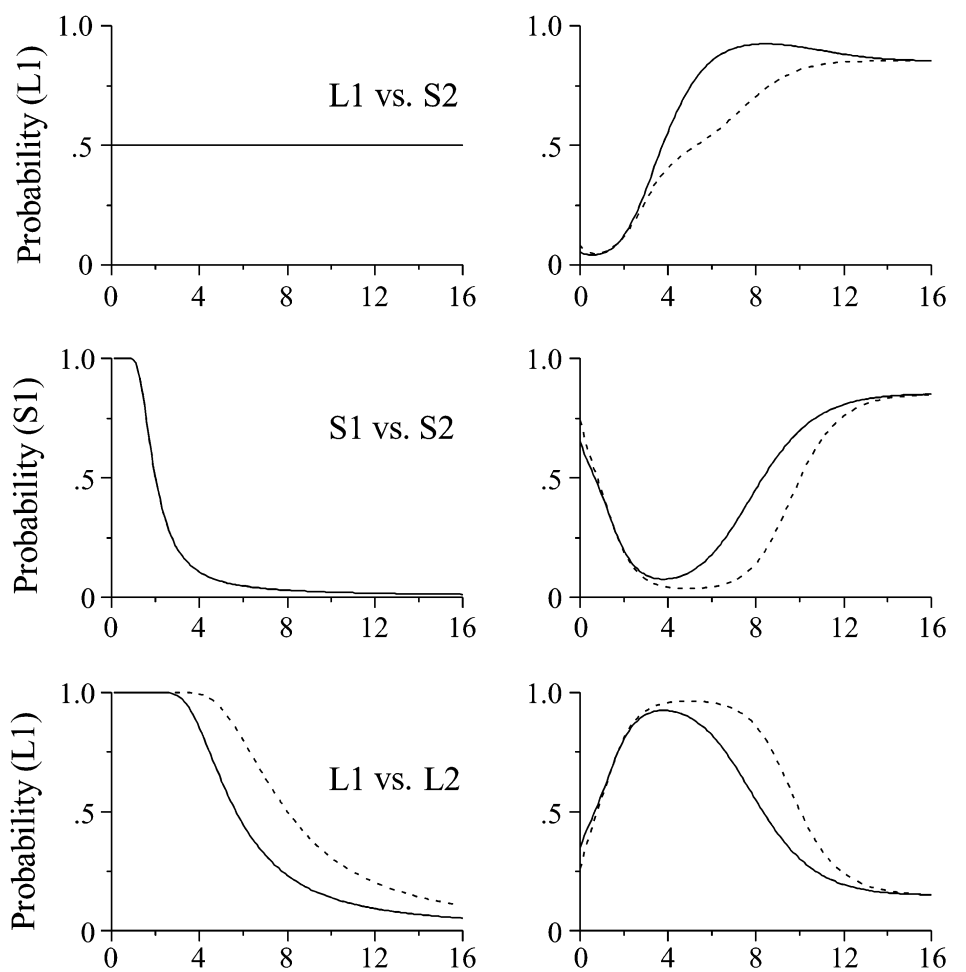

Duration of test stimulus (sec)

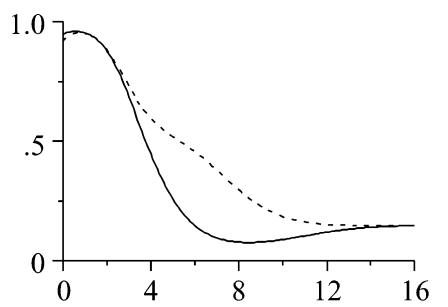

Duration of test stimulus (sec)

Figure 4. Predictions of the scalar expectancy theory (SET) model and the learning-to-time (LeT) model for Group 8 and Group 16. The former learned the discriminations " 1 versus 4 " and " 4 versus 8"; the latter learned the discriminations " 1 versus 4" and " 4 versus 16." In the SET curves, one parameter is used: $\gamma=0.4$. In the LeT curves, three parameters are used: $\lambda=2, \alpha=1$, and $\delta=3.5$.

quently LeT predicts a strong preference for $\mathrm{L} 1$ at $8 \mathrm{sec}$. However, in Group 16 the same degree of uncoupling with S2, and therefore the same preference for L1, must wait for the states most active at $16 \mathrm{sec}$. Hence, LeT predicts that the preference for L1 will grow with test duration more rapidly in Group 8 than in Group 16.

In summary, with the exception of the case of S1 versus L2, the two models predict psychometric functions with very different shapes during the test trials. Moreover, whereas SET predicts no differences between the two groups in the cases of $\mathrm{L} 1$ versus $\mathrm{S} 2$ and $\mathrm{S} 1$ versus $\mathrm{S} 2$, LeT predicts differences between the two groups in all four cases. The second goal of the present study was to test these new predictions.

\section{METHOD}

\section{Subjects}

The subjects were 6 naive pigeons (Columba livia) maintained at $80 \%$ of their free-feeding body weights.

\section{Apparatus}

Two standard chambers for pigeons from Med Associates ${ }^{\circledR}$ (Georgia, VT) were used. The front panel of each chamber contained three keys $2 \mathrm{~cm}$ in diameter, centered on the wall $22 \mathrm{~cm}$ above the floor and $8 \mathrm{~cm}$ apart, center to center. The keys could be illuminated from behind with a red, green, blue, yellow, or white light. Directly below the center key and $4 \mathrm{~cm}$ above the floor was a hopper opening measuring $6 \times 7 \mathrm{~cm}$. The bird had access to mixed grain when the hopper was raised and illuminated with a $7.5-\mathrm{W}$ white light. On the back of the chamber, another 7.5-W houselight 
provided general illumination. An outer box equipped with a ventilating fan enclosed the experimental chamber. A personal computer controlled all experimental events and recorded the data.

\section{Procedure}

Half of the animals, selected at random, learned the discriminations " 1 versus 4" and " 4 versus 8 " (Group 8 ) and the other half learned the discriminations " 1 versus 4" and "4 versus 16" (Group 16). For half of the birds ( 2 from Group 8 and 1 from Group 16), the keylight color assignments were $\mathrm{S} 1=$ red, $\mathrm{L} 1=$ green, $\mathrm{S} 2=$ blue, and L2 = yellow; for the other half ( 1 from Group 8 and 2 from Group 16) they were $\mathrm{S} 1=$ yellow, $\mathrm{L} 1=$ blue, $\mathrm{S} 2=$ green, and $\mathrm{L} 2=$ red. Note that the 4-sec signal was always associated with either the color green or the color blue. Although the color assignments were (partially) counterbalanced, for clarity we describe the procedure and the experimental results as if all of the birds had had the assignment shown in the top panel of Figure 2.

Pretraining. The experiment was divided into three phases: pretraining, training, and testing. During pretraining, the animals first learned to peck the keys through autoshaping and then learned one of the basic discriminations. Each session was divided into trials, and the following sequence of events took place during each trial: The houselight and the center key were illuminated with white light for the duration of the signal (e.g., $4 \mathrm{sec}$ ). Afterward, the center key light was turned off and the side keys were illuminated with different colors (e.g., red and green). A peck at a choice key turned all key lights and the houselight off, and if the choice was correct, it activated the hopper. The hopper duration varied across birds from 2.5 to $5 \mathrm{sec}$ in order to maintain body weight with minimal extrasession feedings. After feeding, a 20 -sec intertrial interval followed. If the choice was incorrect, the intertrial interval started immediately and the trial was repeated (correction method). Sessions ended after the bird had obtained 60 reinforcers.

After the birds learned the discrimination (at least $80 \%$ correct choices, excluding repeated trials, for 5 consecutive sessions), they were trained on the other discrimination. After the second discrimination was learned, the two types of trials alternated across sessions. Because 2 birds from Group 8 (P22 and P28) showed no evidence of learning the "4-versus- 8 " discrimination after 12 sessions, we switched them to the easier discrimination, " 4 versus 16 ," and then gradually shortened the duration of the long signal from 16 to $11.3 \mathrm{sec}$ and, finally, to $8 \mathrm{sec}$. This preliminary training on the two basic discriminations took $22-58$ sessions.

Training. During the first five to seven sessions, half of the trials were Type 1 and half Type 2, correct choices were reinforced, and incorrect choices caused the trial to repeat. Sessions ended after 80 reinforcers ( 40 from each trial type) were obtained. The two colors presented during each trial type always occurred the same number of times on the left and right keys. Then extinction trials were introduced to adapt the birds to the lower rate of food that would be available during the subsequent testing phase. In addition to not ending with food even when a choice was correct, extinction trials were not repeated if the choice was incorrect. Sessions comprised 56 regular trials and 24 extinction trials, for a total of 80 trials. Training lasted until the birds averaged at least $80 \%$ correct choices over five sessions, excluding repeated trials, on each of the four basic signals (range of required sessions: 16-33).

Testing. Each session comprised 56 regular trials and 24 test trials. The difference between regular and test trials was that new pairs of key colors and stimulus durations were presented during the test trials. Specifically, during the regular trials the pairs were always $\mathrm{S} 1-\mathrm{L} 1$ and S2-L2, but during the test trials they were S1-S2, S1-L2, L1-S2, and L1-L2. In addition, the signal durations during the test trials equaled 1,4 , and $8 \mathrm{sec}$ during the first 10 test sessions and 2, 5.7, and $16 \mathrm{sec}$ during the next 10 test sessions (note the logarithmic spacing of the test signal durations). Four new key combinations times three test durations yields 12 different kinds of test tri- als. Each of these trials was presented twice per session, once for each left-key/right-key color assignment, for a total of 20 sessions. To prevent the birds from extinguishing their choices or increasing choice latency in the presence of the new key combinations, half of the test trials (randomly selected) ended with food regardless of the choice made. If during any test session the percentage of a bird's correct choices following one of the four basic signal durations fell below $75 \%$, the bird returned to training (i.e., regular plus extinction trials only) for at least 2 sessions and until its performance was again above $80 \%$. The testing phase, with intercalated training sessions when required, lasted from 20 to 50 sessions across birds.

\section{RESULTS}

During the last five sessions of training, overall proportion correct was consistently high (average for Group $8=.92$; average for Group $16=.95$ ). The range of proportion correct across the four basic durations was $.80-.99$ in Group 8 and .89-.99 in Group 16. An ANOVA on proportion correct, ${ }^{1}$ with group (Group 8 vs. Group 16) as the between-subjects factor and the four signal durations as the within-subjects factor, yielded nonsignificant effects for group $[F(1,4)=0.73]$, duration $[F(3,12)=$ $2.4, p=.12]$, and their interaction $[F(3,12)=.70]$. We conclude that the difference in proportion correct between the two groups and those among the four stimulus durations were small and nonsignificant.

The analysis of the data from the test trials shed light on two questions: (1) Did Group 16 reproduce the results obtained in Machado and Keen's (1999) study, and (2) did the two groups differ? With regard to the first question, Figure 5 shows the average data from Group 16 and the average data from Machado and Keen's study for each of the four key combinations. In general, the shapes of the functions as well as the specific values of preference for the S1 key (top) or the L1 key (bottom) for any given stimulus duration were similar. In particular, the two critical data sets for the choice between L1 and S2 yielded the same monotonically increasing function predicted by LeT but not by SET. In conclusion, despite the fact that $50 \%$ of the test trials were nondifferentially reinforced in the present experiment whereas all of the test trials were conducted in extinction in Machado and Keen's study, their results were fully reproduced.

With regard to the second question, Figure 6 represents the individual and average data from the two groups when the choices were S1 versus L2 and L1 versus S2. The top left and middle panels show that as stimulus duration increased, the preference for $\mathrm{S} 1$ decreased, and more rapidly in Group 8 than in Group 16. This result is shown more clearly in the "average" panel. An ANOVA yielded significant effects of group $[F(1,4)=$ $9.31, p=.04]$, of duration of test stimulus $[F(5,20)=$ $77.4, p<.001]$, and, more important, of their interaction $[F(5,20)=3.97, p=.01]$.

Consider now the bottom panel of Figure 6. The left and middle panels show that as stimulus duration increased, the preference for L1 increased, and more rapidly in Group 8 than in Group 16. Note in particular 

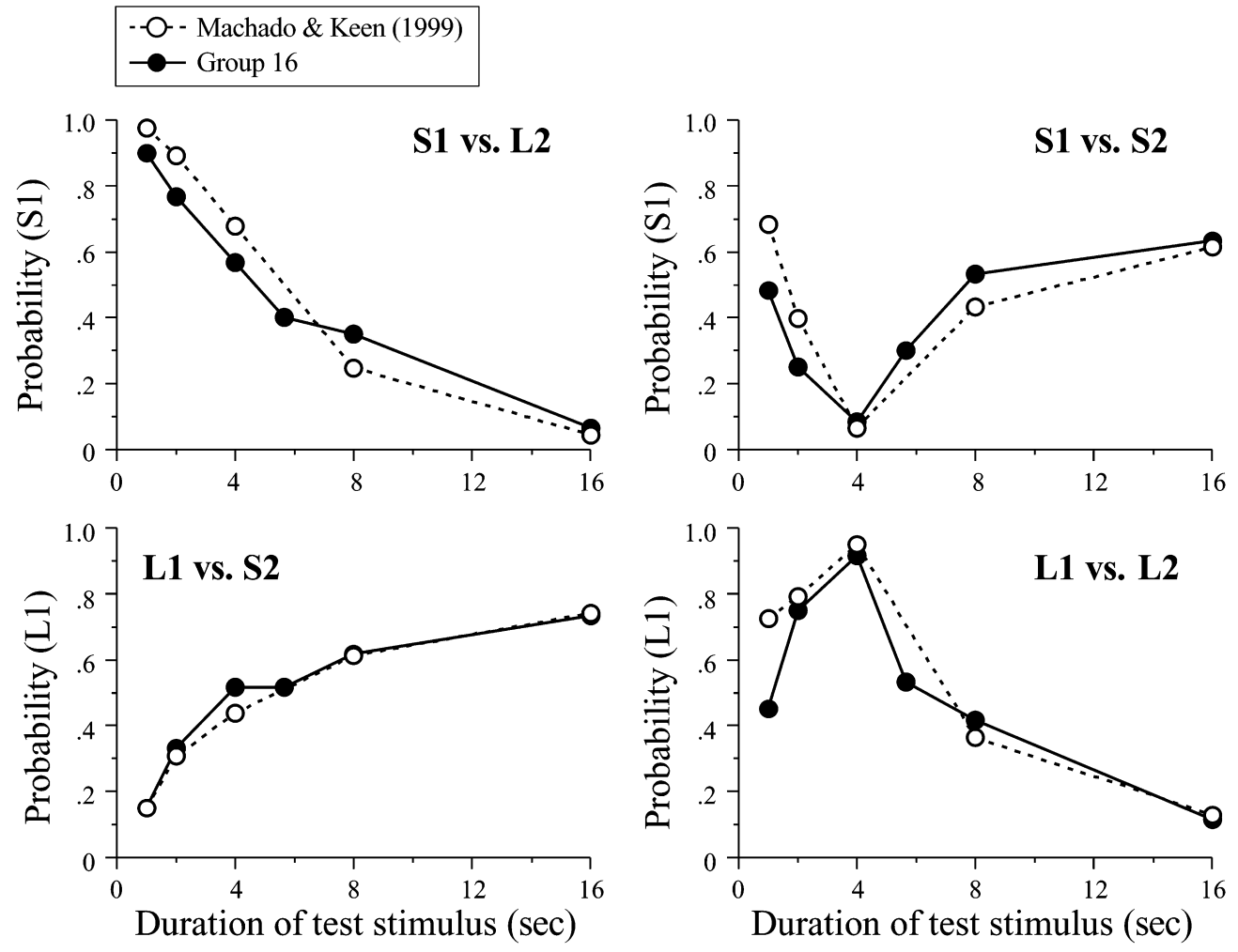

Figure 5. Average data from Group 16 in comparison with the average data from Machado and Keen's (1999) study. Each panel represents one of the four key combinations used during testing. The top and bottom panels show preference for the S1 key and the L1 key, respectively.

that when the test signal equaled $8 \mathrm{sec}$, the birds in Group 8 always chose the L1 key, whereas the birds in Group 16 chose that key only on $50-75 \%$ of the trials. A similar difference occurred at $16 \mathrm{sec}$ : The 3 birds from Group 8 always chose L1, but 2 birds from Group 16 chose $\mathrm{L} 1$ only on $55 \%$ and $65 \%$ of the trials, respectively. The faster rate of increase for Group 8 is made clearer in the "average" data. An ANOVA yielded a nonsignificant effect of group $[F(1,4)=2.12, p=.22]$ and significant effects of duration $[F(5,20)=58.8, p<.001]$ and, more important, of their interaction $[F(5,20)=4.91, p=.005]$.

Figure 7 shows the remainder of the data: the preference for $\mathrm{S} 1$ when the choice was between $\mathrm{S} 1$ and $\mathrm{S} 2$, and the preference for L1 when the choice was between L1 and L2. Concerning the preference for S1 (top panel), the data showed U-shaped curves but no substantial differences between the two groups - the average curves overlapped considerably. An ANOVA yielded only a significant effect of duration $[F(5,20)=9.30, p<.001$; $F(1,4)=.05$ for group and $F(5,20)=.08$ for the interaction]. Concerning the preference for L1 (bottom panel), the obtained functions had a similar, inverted $U$ shape, but the ascending and descending limbs of the function for Group 16 tended to be steeper than the corresponding limbs of the function for Group 8 (see average curves). However, an ANOVA yielded only a significant effect of duration $[F(5,20)=16.5, p<.001 ; F(1,4)=3.21, p=.15$ for group and $F(5,20)=1.14, p=.25$ for the interaction]. In the left and middle panels of Figures 6 and 7, the solid and dotted lines replot LeT's and SET's predictions, respectively. In all cases, the shapes of LeT's predicted curves were similar to those of the corresponding obtained curves. In addition, in most cases the specific predicted values of preference for S1 or L1 also were reasonably close to the specific observed values. The major discrepancy between the predictions of LeT and the obtained data occurred in Group 16 (see Figure 7), for in each case the model predicted curves considerably wider than those that were observed. In fact, as Figure 4 shows, LeT always predicts narrower curves for Group 8 than for Group 16, a prediction at odds with the data.

Concerning SET, the shapes of its predicted curves agreed with the data reasonably well when the choice was between S1 and L2 (see Figure 6, top panel). However, in the other three cases, the shapes of SET's curves were severely at odds with the shapes of the corresponding obtained curves. The discrepancy is particularly noticeable in the case L1 versus S2 (Figure 6, bottom panel).

Another strong prediction of LeT concerns the symmetry of the two pairs of preference curves (Pair 1, S1 vs. L2 and L1 vs. S2; Pair 2, S1 vs. S2 and L1 vs. L2) with respect to the $y=.5$ horizontal line. To determine 

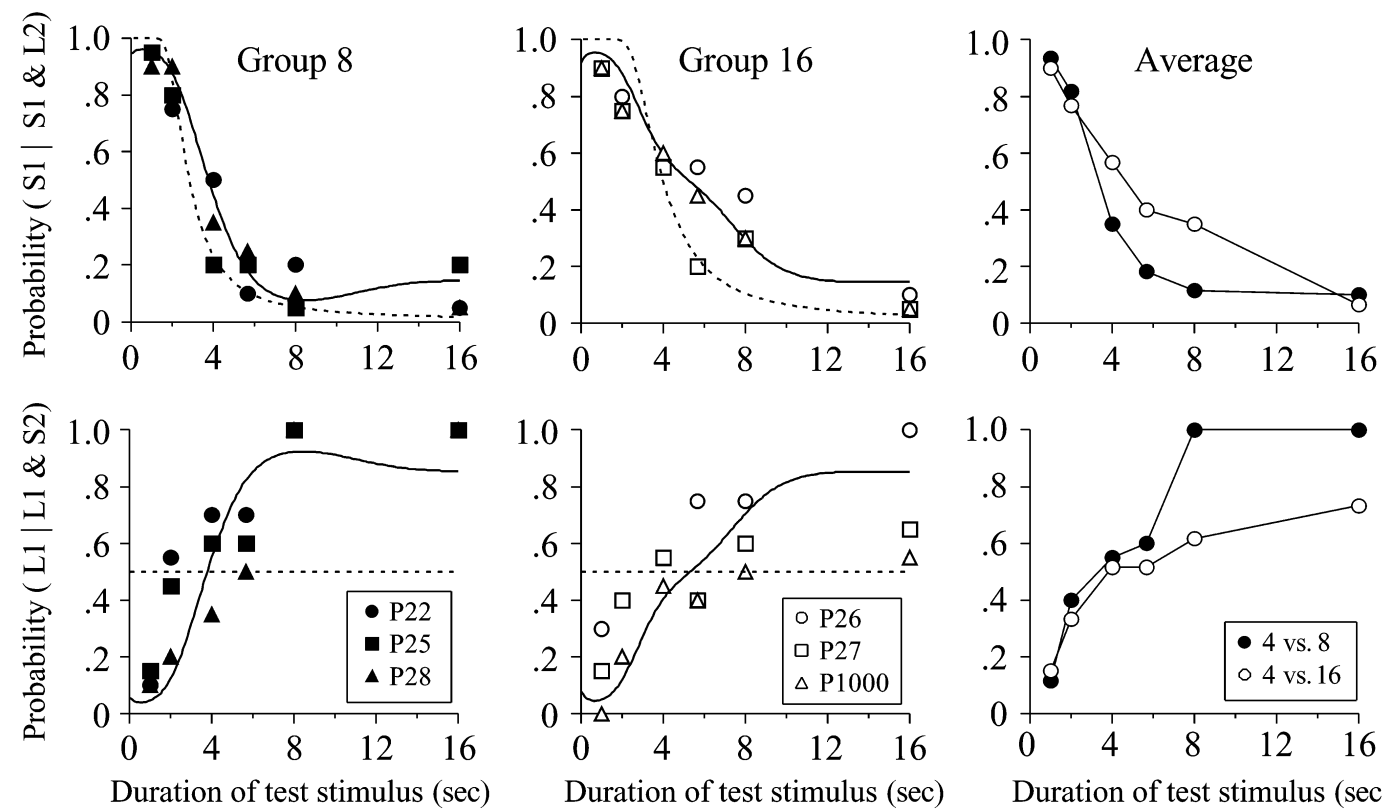

Figure 6. Individual and average data from the two groups when the choices were between S1 and L2 (top) and between $L 1$ and S2 (bottom). In the left and middle panels, the solid and dotted lines show LeT's and SET's predictions, respectively.

whether the data agree with this prediction, in Figure 8 the average data is replotted with one of the curves from each pair reflected along the symmetry line (i.e., the data from L1 vs. S2 and those from L1 vs. L2 were transformed according to the equation "new value $=1-$ original value"). If the symmetry prediction were correct, the two curves in each panel of Figure 8 should overlap. The results are mixed. On the one hand, in each of the six pairs of curves the two members have very similar shapes, but on the other hand, there is some suggestion that the slopes of some descending curves (see the data for Group 16 and for Machado \& Keen, 1999, in the top panel of Figure 8) or the width of the U-shaped curves may differ between the two members of each pair
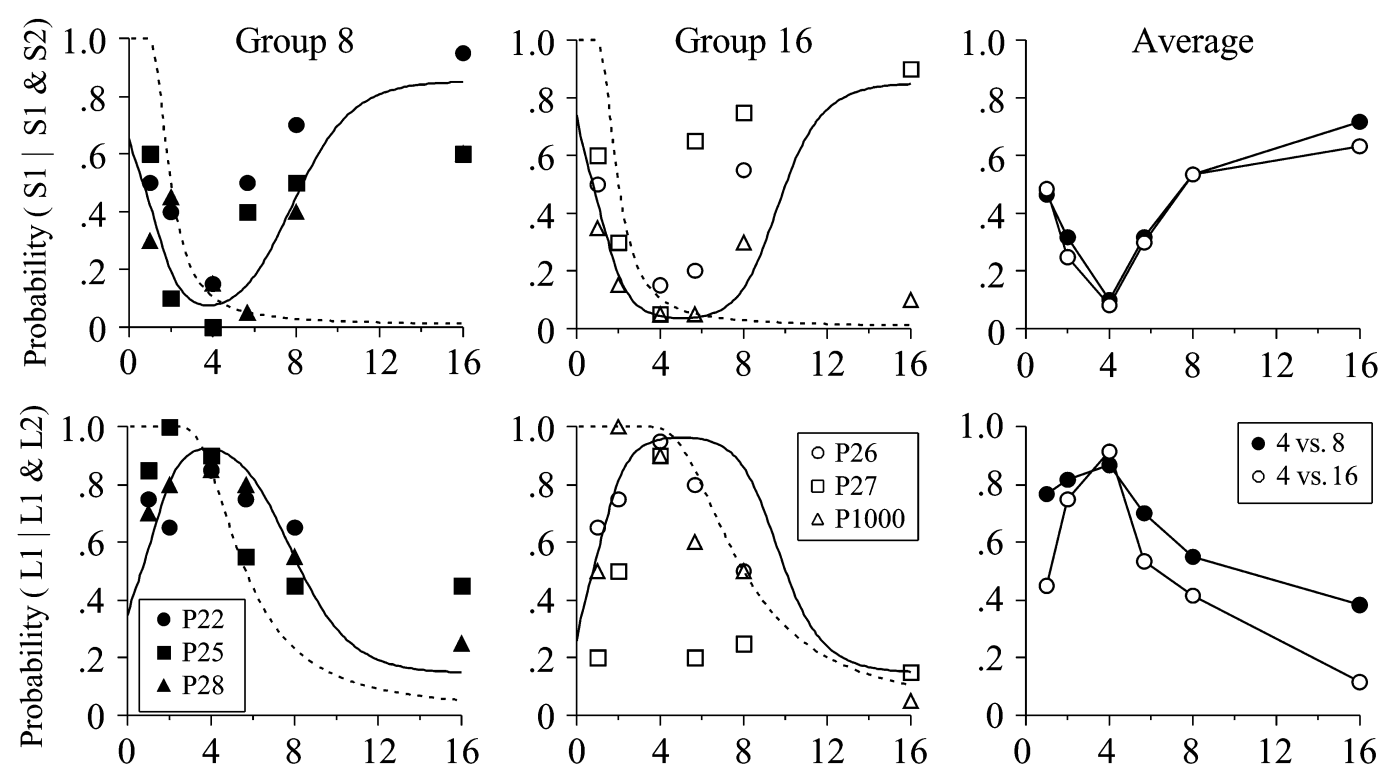

Duration of test stimulus (sec)

Duration of test stimulus (sec)

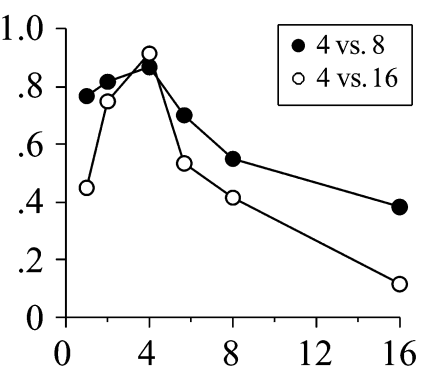

Duration of test stimulus (sec)

Figure 7. Individual and average data from the two groups when the choices were between S1 and S2 (top) and between $L 1$ and $L 2$ (bottom). In the left and middle panels, the solid and dotted lines show LeT's and SET's predictions, respectively. 
Group 8
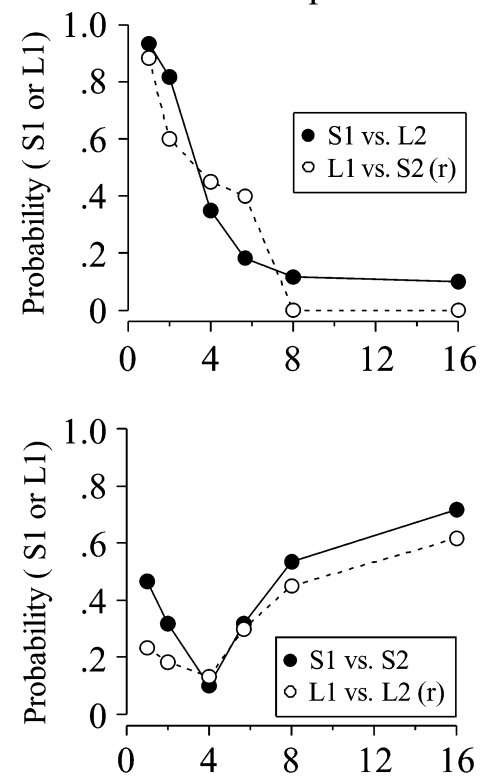

Duration of test stimulus (sec)
Group 16
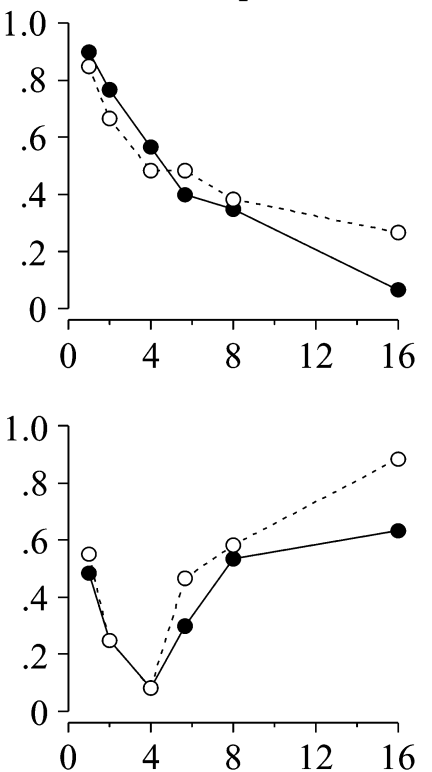

Duration of test stimulus (sec)
Machado \& Keen (1999)
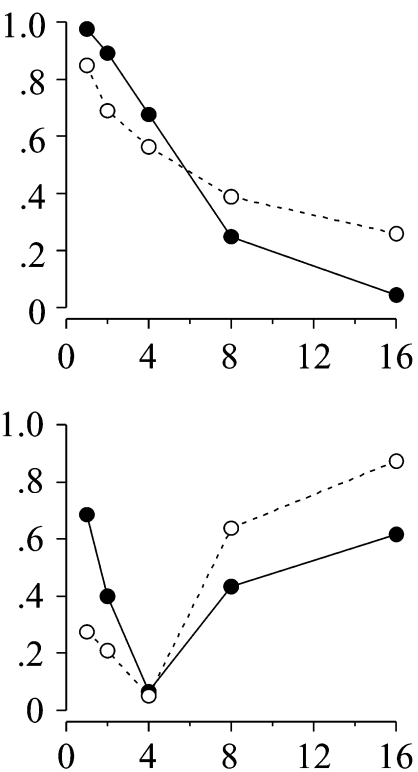

Duration of test stimulus (sec)

Figure 8. Testing for symmetry. The data from L1 versus $S 2$ and $L 1$ versus $L 2$ choices were reflected along the line $y=.5$. LeT predicts superposition of the two curves of each pair.

(see the bottom panel). No stronger conclusions concerning symmetry seem possible at this time.

\section{DISCUSSION}

Six pigeons were exposed to two types of trials. During one type, they learned to choose a red key (S1) after a 1-sec signal and a green key (L1) after a 4-sec signal; during another type of trial, they learned to choose a blue key (S2) after a 4-sec signal and a yellow key (L2) after an 8-sec (Group 8) or a 16-sec (Group 16) signal. After the two basic discriminations were learned, the birds were exposed to a set of tests in which new key combinations were introduced and preference for a particular key was assessed as a function of signal duration. In the most critical test, the birds chose between two keys that had been associated with the same reinforced duration (i.e., 4 sec for both L1 and S2).

The results from Group 16 reproduced Machado and Keen's (1999) earlier findings. Thus, when the choice was between L1 and S2, preference for L1 increased with the duration of the test signal, a result consistent with LeT but not with SET. The functions obtained with the other three key combinations also reproduced the functions obtained in the previous study and, once again, the observed pattern of preferences was closer to LeT's predictions than to those of SET.

LeT predicted distinctive curves for Groups 8 and 16. In particular, the model predicted that preference for L1 over S2 would increase with stimulus duration for both groups, but would do so faster for Group 8 than for
Group 16. This result was observed and, again, it is at odds with the predictions of SET. Similarly, LeT predicted that preference for S1 over L2 would decrease with stimulus duration for both groups, but would do so faster for Group 8 than for Group 16. This result was observed, although in this case it is consistent with the predictions of both LeT and SET. Concerning the cases of $\mathrm{S} 1$ versus $\mathrm{S} 2$ and $\mathrm{L} 1$ versus L2, the general shape of the observed curves were consistent with those predicted by LeT, but the prediction of narrower curves for Group 8 than for Group 16 was not borne out. Finally, we note also that the two pairs of curves-S1-L2 and L1-S2 on the one hand, and S1-S2 and L1-L2 on the other-were reasonably symmetric along the $y=.5$ line.

The foregoing comparison between the two models may be seen as somewhat "unfair" because only one parameter is used in SET's predictions whereas three are used in LeT's predictions. Aware of this fact, we have stressed throughout this article the shape and trend of the curves and not their specific values, for whereas the latter change with the parameters the former depend primarily on the structure of the model. Consider again the critical finding that, when a choice must be made between L1 and S2, preference for L1 increased with signal duration. SET fails to predict this finding because it assumes independence between the memory stores formed during training. That is, the contents of the "short" memory store depend only on the duration of the short signal; they do not depend on the duration of the long signal. Independence of memory stores means that SET has no mechanism to accommodate the contextual effects re- 
vealed in the data: the fact that when a pigeon was given a choice between L1 and S2, its behavior depended on the durations associated with S1 and L2, neither of which was present at the time of the choice! SET's limitation is not parametric but structural-in principle, it cannot deal with this finding (see also Machado \& Guilhardi, 2000).

In contrast, two structural features of LeT allow it to account for the contextual effects. First, the effects of learning are represented by the couplings between the behavioral states and an operant response. Because some couplings may be strong whereas others may be weak, the strength of the operant response may vary with signal duration (e.g., response L1 will be weak at $1 \mathrm{sec}$ but strong at $4 \mathrm{sec}$ ). Second, according to LeT's learning rule, when the couplings between the behavioral states and one response change in one direction (up with reinforcement, down with extinction), the couplings between the behavioral states and the other response change by the same amount but in the opposite direction (e.g., the couplings between the initial states and $\mathrm{S} 1$ will be strong, but the couplings between those states and L1 will be weak). These two structural features enable the model to predict that the strengths of the responses L1 and S2 will depend on the duration of the test signal and on the durations associated with S1 and L2, the context effect.

Machado and Keen (1999) also reported that 6 of their 8 pigeons displayed time-differentiated behavioral patterns during the signal, patterns that were strongly correlated with choice performance during the test trials. Similar patterns were observed during the present experiment by most but not all of the birds. To illustrate, Pigeon P27 (Group 16) typically started the trial facing the intelligence panel; 1 or $2 \mathrm{sec}$ into the trial, the bird rotated its body $60^{\circ}$ to the right and started to peck the floor; at $4-6 \mathrm{sec}$ it started to move, often turning $360^{\circ}$; at $8-10 \mathrm{sec}$ it moved to the left side of the intelligence panel and pecked at the top left corner of the box until the end of the signal. Thus, this bird's behavior early into the signal (pecking the floor on the right side of the box) contrasted sharply with its behavior late into the signal (pecking the top left corner of the box).

Pigeon P28 (Group 8) also started the trial facing the key panel. It then moved to the right side of that panel and, 2 or $3 \mathrm{sec}$ into the trial, started to oscillate its head as if trying to see the other keys. These oscillations were accompanied by lateral movements that took the bird from the right side of the box to the left side and back; at 6 or $7 \mathrm{sec}$ it lowered its head and started to peck the region of the intelligence panel between the center key and the feeder.

Pigeon P25 from Group 8 displayed a different type of pattern. This bird approached the center key, pecked it once or so during the first $3 \mathrm{sec}$, and then, as the signal elapsed, pecked the key at an increasing rate. In contrast, Pigeon P26 simply moved in the box with no apparent spatial regularity. Why do some birds display stereotypical patterns whereas others do not? How are these patterns acquired? And what role do they play in temporal discriminations? These are some of the questions that remain to be answered (also see Fetterman, Killeen, \& Hall, 1998; Richelle \& Lejeune, 1980).

Finally, we note that our key findings - that is, that in a choice of L1 versus S2 (1) preference for L1 increases monotonically with stimulus duration and (2) that increase takes place more rapidly for Group 8 than Group 16-may be seen as the results of peak shift in the temporal domain. Pecking of the L1 and S2 keys is an operant controlled by the duration of the signal. This control is maximal at $4 \mathrm{sec}$ (the discriminative stimulus, or $\mathrm{S}^{\mathrm{D}}$ ), and, as in any other generalization gradient, it decreases as the signal duration departs from the $\mathrm{S}^{\mathrm{D}}$. In Group 8, the 8-sec signal (associated with key L2) may be seen as an $\mathrm{S}^{\Delta}$ for pecking the $S 2 \mathrm{key}$. If we assume that the effect of the $\mathrm{S}^{\Delta}$ is to shift the peak of the generalization gradient away from the $\mathrm{S}^{\Delta}$, then the gradient for $\mathrm{S} 2$ would have its maximum below $4 \mathrm{sec}$. By similar reasoning, we would expect the peak of the generalization gradient for $\mathrm{L} 1$ to shift to the right because its $\mathrm{S}^{\Delta}$ is at 1 sec. The net effect of these two shifts is that the gradient for S2 would be to the left of the gradient for L1, which could explain why preference for L1 over S2 increases with test duration. Furthermore, if the magnitude of the shift increases as the $\mathrm{S}^{\Delta}$ approaches the $\mathrm{S}^{\mathrm{D}}$, then the peak of the generalization gradient associated with S2 would be smaller in Group $8\left(\mathrm{~S}^{\Delta}-\mathrm{S}^{\mathrm{D}}=4\right)$ than in Group 16 $\left(S^{\Delta}-S^{D}=12\right)$. Perhaps this difference could explain the faster rate of increase of the preference for L1 in Group 8.

\section{REFERENCES}

CATANiA, A. C. (1970). Reinforcement schedules and the psychophysical judgments: A study of some temporal properties of behavior. In W. N. Schoenfeld (Ed.), The theory of reinforcement schedules (pp. 142). New York: Appleton-Century-Crofts.

Church, R. M., \& Deluty, M. Z. (1977). Bisection of temporal intervals. Journal of Experimental Psychology: Animal Behavior Processes, 3, 216-228.

FetTERMAN, J. G., \& Killeen, P. R. (1991). Adjusting the pacemaker. Learning \& Motivation, 22, 226-252.

Fetterman, J. G., Killeen, P. R., \& Hall, S. (1998). Watching the clock. Behavioral Processes, 44, 211-224.

Gallistel, C. R. (1990). The organization of learning. Cambridge, MA: MIT Press/Bradford Books.

Gallistel, C. R., \& GibBon, J. (2002). The symbolic foundations of conditioned behavior. Mahwah, NJ: Erlbaum.

GibBon, J. (1977). Scalar expectancy theory and Weber's law in animal timing. Psychological Review, 84, 279-325.

GibBon, J. (1981). On the form and location of the psychometric bisection function for time. Journal of Mathematical Psychology, 24, 58-87.

GiBBON, J. (1991). Origins of scalar timing theory. Learning \& Motivation, 22, 3-38.

KILlEEN, P. R., \& FetTerman, J. G. (1988). A behavioral theory of timing. Psychological Review, 95, 274-285.

Machado, A. (1997). Learning the temporal dynamics of behavior. Psychological Review, 104, 241-265.

MACHADO, A., \& GUILHARDI, P. (2000). Shifts in the psychometric function and their implications for models of timing. Journal of the Experimental Analysis of Behavior, 74, 25-54.

MACHADO, A., \& KEEN, R. (1999). Learning to time (LET) or scalar expectancy theory (SET)? A critical test of two models of timing. Psychological Science, 10, 285-290.

PlATt, J. R., \& DaVIS, E. R. (1983). Bisection of temporal intervals by 
pigeons. Journal of Experimental Psychology: Animal Behavior Processes, $9,160-170$.

RiCHELle, M., \& LEJEUne, H. (1980). Time in animal behavior. Oxford: Pergamon.

Shettleworth, S. (1998). Cognition, evolution, and behavior. New York: Oxford University Press

Staddon, J. E. R. (2001). Adaptive dynamics. Cambridge, MA: MIT Press.
StubBs, D. A. (1968). The discrimination of stimulus duration by pigeons. Journal of the Experimental Analysis of Behavior, 11, 223-238.

\section{NOTE}

1. In this and all subsequent ANOVAs, an arcsine transformation of proportion correct prior to the statistical analysis always led to the same conclusions.

\section{APPENDIX}

In what follows, we assume that the two signals occur with equal probability (.5) and that the correct choices following the short (S) and long (L) signals are the red (R) and green $(\mathrm{G})$ keys, respectively.

\section{LeT-Simple Bisection}

LeT has three components: the behavioral states, their couplings with the instrumental responses, and the strength of the instrumental responses (see Figure 1). According to the model, the onset of the training stimulus triggers the transitions across the behavioral states. When the stimulus ends, the subject responds S1 or L1. If the response is reinforced, then the association between each state and the choice response increases and the association between each state and the other response decreases by the same amount; if the choice response is incorrect, then the association between that response and each state decreases and the association between the other response and each state increases by the same amount.

To obtain LeT's predictions (see Machado, 1997, for details) we need the expression for the activation of state $n$ after a signal of $t \mathrm{sec}, X(t, n)$, and the expressions for the couplings between state $n$ and the responses $\mathrm{R}$ and $\mathrm{G}, W \mathrm{R}(n)$ and $W \mathrm{G}(n)$, respectively. In LeT, $X(t, n)$ follows the Poisson distribution, a minimal, parsimonious assumption:

$$
X(n, t)=\frac{\exp (-\lambda t)(\lambda t)^{n}}{n !}
$$

where $\lambda>0$ is a parameter that controls how fast the activation spreads across the states.

During training, the changes in $W \mathrm{R}(n)$ and $W \mathrm{G}(n)$ are always symmetric: If $W \mathrm{R}(n)$ increases because response $\mathrm{R}$ was reinforced or decreases because response $\mathrm{R}$ was extinguished, then $W \mathrm{G}(n)$ changes by the same amount but in the opposite direction. The same is true when response $\mathrm{G}$ occurs and $W \mathrm{G}(n)$ changes; $W \mathrm{R}(n)$ also changes by the same amount but in the opposite direction. Because initially there is no bias, both $W \mathrm{R}(n)$ and $W \mathrm{G}(n)$ start at .5 . It follows that $W \mathrm{G}(n)$ will always equal $1-W \mathrm{R}(n)$.

Next, we show how to derive the value of $W \mathrm{R}(n)$ after $m$ trials. We start with LeT's equation for the expected value of $\Delta W \mathrm{R}(n)$ after one trial (see Machado, 1997, p. 256). If the short stimulus occurs, then $\Delta W R(n)$ increases in proportion to the activation of the state, $X(\mathrm{~S}, n)$, and the difference $[1-W \mathrm{R}(n)]$ :

$$
\Delta W \mathrm{R}(n)=\alpha X(\mathrm{~S}, n)[1-W \mathrm{R}(n)] .
$$

Parameter $\alpha>0$ is a learning rate parameter. If the long stimulus occurs, then $\Delta W \mathrm{R}(n)$ decreases in proportion to the activation of the state, $X(\mathrm{~L}, n)$, and the current value of $W \mathrm{R}(n)$ :

$$
\Delta W \mathrm{R}(n)=-\alpha X(\mathrm{~L}, n) W \mathrm{R}(n) .
$$

The expected value of $\Delta W \mathrm{R}(n)$ is therefore equal to

$$
\mathrm{E}[\Delta W \mathrm{R}(n)]=\frac{1}{2} \alpha X(\mathrm{~S}, n)[1-W \mathrm{R}(n)]-\frac{1}{2} \alpha X(\mathrm{~L}, n) W \mathrm{R}(n) .
$$

Equation 2 may be expressed as a linear difference equation relating the expected value of $W \mathrm{R}(n)$ on trial $m+1$, which we designate by $\mathrm{E}\left[W \mathrm{R}(n)_{m+1}\right]$, to the expected value of $W \mathrm{R}(n)$ on trial $m$, designated $\mathrm{E}\left[W \mathrm{R}(n)_{m}\right]$ :

$$
\mathrm{E}\left[W \mathrm{R}(n)_{m+1}\right]=\frac{1}{2} \alpha X(\mathrm{~S}, n)+\left\{1-\frac{1}{2} \alpha[X(\mathrm{~S}, n)+X(\mathrm{~L}, n)]\right\} \mathrm{E}\left[W \mathrm{R}(n)_{m}\right]
$$

Equation 3 has the solution

$$
\mathrm{E}\left[W \mathrm{R}(n)_{m}\right]=\frac{X(\mathrm{~S}, n)}{X(\mathrm{~S}, n)+X(\mathrm{~L}, n)}-\left(1-\alpha \frac{X(\mathrm{~S}, n)+X(\mathrm{~L}, n)}{2}\right)^{m}\left(\frac{X(\mathrm{~S}, n)}{X(\mathrm{~S}, n)+X(\mathrm{~L}, n)}-W \mathrm{R}(n)_{0}\right),
$$

where $W \mathrm{R}(n)_{0}$ is the initial value of $W \mathrm{R}(n)$. The corresponding expression for $W \mathrm{G}(n) m$ is

$$
\mathrm{E}\left[W \mathrm{G}(n)_{m}\right]=1-\mathrm{E}\left[W \mathrm{R}(n)_{m}\right] .
$$


From the expressions for $X(t, n), W \mathrm{R}(n)$, and $W \mathrm{G}(n)$, we get the strengths of the $\mathrm{R}$ and $\mathrm{G}$ responses $(\mathrm{RR}(t)$ and $\mathrm{RG}(t)$, respectively) after a signal of duration $t$ :

$$
\begin{aligned}
& \operatorname{RR}(t)=\sum_{n} X(t, n) W \mathrm{R}(n)_{m} \\
& \operatorname{RG}(t)=\sum_{n} X(t, n) W \mathrm{G}(n)_{m} .
\end{aligned}
$$

Finally, the probability of choosing the G key at time $t$ equals

$$
\begin{aligned}
P(t) & =\frac{\exp [\delta \times \mathrm{RG}(t)]}{\exp [\delta \times \mathrm{RR}(t)]+\exp [\delta \times \mathrm{RG}(t)]} \\
& =\frac{1}{1+\exp \{-\delta[1-2 \mathrm{RR}(t)]\}}
\end{aligned}
$$

(see Machado, 1997), where $\delta>0$ is a sensitivity parameter. In the last equality, we have used the fact that $\mathrm{RG}(t)=1-\mathrm{RR}(t)$.

In summary, LeT's predictions are based on Equation 1, which describes the temporal activation of the behavioral states and uses parameter $\lambda$; Equations 4 and 5 , which describe the effects of learning and use parameter $\alpha$; and Equation 7, which yields choice probability on the basis of the two response strengths given by Equation 6 and uses parameter $\delta$.

The curves shown in the text were obtained assuming that $W \mathrm{R}(n)_{0}=W \mathrm{G}(n)_{0}=.5, m=3,000$ trials $(60$ sessions of 50 trials each), $\lambda=2, \alpha=1$, and $\delta=3.5$.

\section{LeT-Double Bisection}

Assume now that red and green are associated with 1-sec and 4-sec signals, respectively, and that blue and yellow are associated with 4-sec and 16-sec signals, respectively. During testing, when two novel keys (e.g., $\mathrm{L} 1$ and S2) are presented, we derive LeT's prediction by (1) computing $W \mathrm{G}(n)$ and $W \mathrm{~B}(n)$ with Equation 4 , noting that $\mathrm{S}=1 \mathrm{sec}$ and $\mathrm{L}=4 \mathrm{sec}$ for $\mathrm{G}$, but $\mathrm{S}=4 \mathrm{sec}$ and $\mathrm{L}=16$ for $\mathrm{B}$; (2) inserting these values into Equation 6 to obtain $\mathrm{RG}(t)$ and $\mathrm{RB}(t)$; and, finally, (3) inserting $\mathrm{RG}(t)$ and $\mathrm{RB}(t)$ into Equation 7 .

\section{SET}

The curves predicted by SET were obtained from Equations 16 and 45 in Gibbon (1981). The model's single parameter, $\gamma$, is the coefficient of variation. To draw the curves in the text, we assumed $\gamma=0.4$. The probability of choosing the R key (associated with the short signal, $\mathrm{S}$ ) after a $t$-sec signal when the choice is from $\mathrm{R}$ and the $\mathrm{G}$ key (associated with the long signal, L), for example, equals

$$
P(t)=\int_{-\infty}^{\mathrm{C} 1(t)} N(0,1) d \tau-\int_{-\infty}^{\mathrm{C} 2(t)} N(0,1) d \tau,
$$

where

$$
\begin{aligned}
& \mathrm{C} 1(t)=\frac{1}{\gamma}\left(\frac{\sqrt{\mathrm{S} \times \mathrm{L}}}{t}-1\right), \\
& \mathrm{C} 2(t)=-\frac{1}{\gamma}\left(\frac{\sqrt{\mathrm{S} \times \mathrm{L}}}{t}+1\right),
\end{aligned}
$$

and $N(0,1)$ is the reduced normal density function

$$
\frac{1}{\sqrt{2 \pi}} \exp \left(-\frac{\tau^{2}}{2}\right)
$$

(Manuscript received July 8, 2004;

revision accepted for publication September 28, 2004.) 